Pacific Journal of Mathematics

FOLIATION BY CONSTANT MEAN CURVATURE SPHERES 


\title{
FOLIATION BY CONSTANT MEAN CURVATURE SPHERES
}

\author{
RUgANG YE
}

\begin{abstract}
Let $M$ be a Riemannian manifold of dimension $n+1$ and $p \in M$. Geodesic spheres around $p$ of small radius constitute a smooth foliation. We shall show that this foliation can be perturbed into a foliation whose leaves are spheres of constant mean curvature, provided that $p$ is a nondegenerate critical point of the scalar curvature function of $M$. The obtained foliation is actually the unique foliation by constant mean curvature hypersurfaces which is regularly centered at $p$ (Definition 1.1). On the other hand, if $p$ is not a critical point of the scalar curvature function, then there exists no such foliation.
\end{abstract}

0. Introduction. The perturbation procedure consists not only of normal perturbation of geodesic spheres, but also of perturbation of their center, which is used to deal with the kernel of the linearized operator of the perturbation equation. On the other hand, it is crucial to control the magnitude of the center perturbation in order to retain the foliation property. Note that the above mentioned "regularly centered" condition is a natural geometric one, but it appears fairly restrictive. What weaker geometric conditions imply it? Are all foliations by constant mean curvature spheres (or hypersurfaces) which are centered at $p$ (Definition 1.1) automatically regularly centered at $p$ ? We shall treat these problems in a subsequent paper. (The answer to the second question is yes in dimension $n=1$ and "almost" yes in dimension $n=2$.)

The techniques in this paper can be applied to produce foliations by constant mean curvature spheres on asymptotically flat manifolds of nonzero mass.

Part of this work was done while the author was visiting the University of California, San Diego. We thank S. T. Yau for his kind invitation. We are grateful to him, R. Schoen and G. Huisken for helpful discussions on the subject.

Notation. $O\left(r^{m}\right)=r^{m}$. a smooth function. Throughout the paper we use \|\| to denote the norm of a vector and the specific norm used in each case should be clear from the context. 
1. Perturbation. For convenience, we assume $M \in C^{\infty}$. We refer to [2] and [1] for basic terminology in local Riemannian geometry. Let $S_{r}(q), B_{r}(q)$ denote respectively the geodesic sphere and open geodesic ball of radius $r>0$ and center $q \in M$. Consider a (henceforth fixed) point $p$ and choose an orthonormal basis $\left\{e_{j}\right\}$ for $T_{p} M$. Put $r_{p}=\frac{1}{8}$. the injectivity radius at $p, \mathbb{B}_{r}=\left\{x \in \mathbb{R}^{n+1}:\|x\|<r\right\}$, and $S^{n}=\partial \mathbb{B}_{1}$. For $\tau=\left(\tau^{1}, \ldots, \tau^{n+1}\right) \in \mathbb{R}^{n+1}$ with $\|\tau\|<r_{p}$ we define $\varphi_{\tau}: \mathbb{B}_{2 r_{p}} \rightarrow M$ by

$$
\varphi_{\tau}(x)=\exp _{c(\tau)}\left(x^{i} e_{i}^{\tau}\right),
$$

where $c(\tau)=\exp _{p}\left(\tau^{i} e_{i}\right), e_{i}^{\tau}$ are the parallel transports of $e_{i}$ to $c(\tau)$ along the geodesic $\left.c(t \tau)\right|_{0 \leq t \leq 1}$ and the summation convention is used. For each $\tau, \varphi_{\tau}$ gives rise to a Riemannian normal coordinate system at $c(\tau)$. We put $d s^{2}=$ the metric tensor of $M, g_{i j}^{\tau}(x)=$ the coefficients of $d s^{2}$ in the coordinate $\varphi_{\tau}$ and $g^{\tau}=\operatorname{det}\left(g_{i j}^{\tau}\right)$. Then by a straightforward computation the inward mean curvature function of $S_{\rho}\left(c(\tau)\right.$ ) (pulled back to $\partial \mathbb{B}_{\rho}$ by $\varphi_{\tau}$ ) is given by

$$
h(x)=\frac{n}{\rho}+\frac{\partial}{\partial r} \log \sqrt{g^{\tau}(x)}, \quad x \in \partial \mathbb{B}_{\rho},
$$

where $r=\|x\|$.

This formula together with the following lemma reveals basic relations between the mean curvature of geodesic spheres and the curvature of $M$.

\section{LEMMA 1.1. We have}

$$
\begin{gathered}
g^{\tau}(x)=1-\frac{1}{3} R_{i j}^{\tau}(0) x^{i} x^{j}-\frac{1}{6} R_{i j ; k}^{\tau}(0) x^{i} x^{j} x^{k} \\
-\left(\frac{1}{20} R_{i j ; k l}^{\tau}(0)+\frac{1}{90} R_{p i j m}^{\tau}(0) R_{p k l m}^{\tau}(0)\right. \\
\left.\quad-\frac{1}{18} R_{i j}^{\tau}(0) R_{k l}^{\tau}(0)\right) x^{i} x^{j} x^{k} x^{l} \\
+a_{i j k l m}(\tau, x) x^{i} x^{j} x^{k} x^{l} x^{m},
\end{gathered}
$$

where $R_{i j}^{\tau}, R_{i j ; k}^{\tau}, R_{i j ; k l}^{\tau}, R_{p i j m}^{\tau}$ are respectively the coefficients of $\mathrm{Ric}$ (Ricci tensor), $\nabla$ Ric, $\nabla \nabla$ Ric and Riem (Riemann curvature tensor): in the coordinate $\varphi_{\tau}$ and $a_{i j k l m}(\tau, x)$ denote some smooth functions. We shall omit the superscript $\tau$ for $\tau=0$.

For a proof of this lemma we refer to [2]. 
Let $\nu$ denote the inward unit normal of $S^{n}:=\partial \mathbb{B}_{1}$ and $\alpha_{r}$ the dilation $x \mapsto r x$ for $r>0$. For $\varphi \in C^{2}\left(S^{n}\right)$ we define $S_{\varphi}^{n}=$ $\left\{x+\varphi(x) \nu(x): x \in S^{n}\right\}$ and $S_{r, \tau, \varphi}=\varphi_{\tau}\left(\alpha_{r}\left(S_{\varphi}^{n}\right)\right)$. Note that $S_{r, \tau, 0}=S_{r}(c(\tau))$ and $S_{\varphi}^{n}$ is an embedded $C^{2}$ surface if only $\|\varphi\|_{C^{1}}<\delta_{0}$ for some number $\delta_{0}>0$. Here and in the sequel, unless otherwise stated, we use the standard metric on $S^{n}$. For $0<r<r_{p}$, $\|\tau\|<r_{p},\|\varphi\|_{C^{1}}<\delta_{0}$ and $x \in S^{n}$ we put

$H(r, \tau, \varphi)(x)=$ the inward mean curvature of the surface $S_{\varphi}^{n}$ at $x+\varphi(x) \nu(x)$ w.r.t. the metric $d s_{\tau, r}^{2}$ on $\mathbb{B}_{2}$,

where $d s_{\tau, r}^{2}=r^{-2} \alpha_{r}^{*}\left(\varphi_{\tau}^{*}\left(d s^{2}\right)\right)$. One readily checks that $d s_{\tau, r}^{2}$ extends smoothly to $r=0$ with $d s_{\tau, 0}^{2} \equiv$ the euclidean metric. Hence $H(r, \tau, \varphi)$ also extends to $r=0$. On the other hand, we have

(1.3) $H(r, \tau, \varphi)(x)=r$. the inward mean curvature of

$$
S_{r, \tau, \varphi} \text { at } \varphi_{\tau}(r(x+\varphi(x) \nu(x))) \text {. }
$$

In particular, by (1.1)

$$
H(r, \tau, 0)(x)=n+r \frac{\partial}{\partial r} \log \sqrt{g^{\tau}(r x)},
$$

which implies on account of (1.2)

$$
\text { (1.5) } \begin{aligned}
H(r, \tau, 0)(x)= & n-\frac{1}{3} R_{i j}^{\tau}(0) x^{i} x^{j} r^{2}-\frac{1}{4} R_{i j ; k}^{\tau}(0) x^{i} x^{j} x^{k} r^{3} \\
& +a_{i j k l}(\tau) x^{i} x^{j} x^{k} x^{l} r^{4}+O\left(r^{5}\right)
\end{aligned}
$$

where $a_{i j k l}(\tau)$ denotes some smooth functions.

We consider $H(r, \tau, \cdot)$ as a mapping from $C^{2,1 / 2}\left(S^{n}\right)$ into $C^{0,1 / 2}\left(S^{n}\right)$ and let $H_{\varphi}$ denote the differential of $H$ w.r.t. $\varphi$. Clearly $H_{\varphi}(r, \tau, 0)$ is just the Jacobi operator $\Delta+\|B\|^{2}+\operatorname{Ric}(\nu)$ on $S^{n}$ relative to the metric $d s_{\tau, r}^{2}$, where $B$ denotes the second fundamental form. We indicate the dependence on $d s_{\tau, r}^{2}$ as follows

$$
H_{\varphi}(r, \tau, 0)=\Delta_{r, \tau}+\left\|B_{r, \tau}\right\|^{2}+\operatorname{Ric}_{r, \tau}(\nu) .
$$

It follows that $H_{\varphi}(0, \tau, 0)=L:=\Delta_{S^{n}}+n$, where $\Delta_{S^{n}}$ is the standard Laplace operator on $S^{n}$. The Jacobi operator $L$ has an $(n+1)$ dimensional kernel $K$ consisting of first order spherical harmonics. Correspondingly, we obtain $L_{2}$-decompositions $C^{2,1 / 2}\left(S^{n}\right)=K \oplus K$ and $C^{0,1 / 2}\left(S^{n}\right)=K \oplus L\left(K^{\perp}\right)$. Let $P$ denote the orthogonal projection from $C^{0,1 / 2}\left(S^{n}\right)$ onto $K$ and $T: K \rightarrow \mathbb{R}^{n+1}$ the isomorphism sending $\left.x^{i}\right|_{n}$ to $\mathbf{e}_{i}=$ the $i$ th coordinate basis. Put $\widetilde{P}=T P$. 
LEMMA 1.2. We have

$$
\begin{aligned}
\widetilde{P}(H(r, \tau, \varphi))= & -\frac{\omega_{n+1}}{2(n+3)} r^{3} R_{, l}^{\tau}(0) e_{l} \\
& +\widetilde{P}\left(\int_{0}^{1} H_{\varphi}(r, \tau, t \varphi) \varphi d t\right)+O\left(r^{5}\right),
\end{aligned}
$$

where $\omega_{n+1}=\operatorname{Vol}\left(\mathbb{B}_{1}\right)$ and $R_{, l}^{\tau}$ denotes the derivatives of the scalar curvature function $R$ of $M$ in the coordinate system $\varphi_{\tau}$.

Proof. From (1.5) we deduce

$$
\widetilde{P}(H(r, \tau, 0))=-\frac{1}{4} r^{3} e_{l} R_{i j ; k}^{\tau}(0) \int_{S^{n}} x^{i} x^{j} x^{k} x^{l} d A+O\left(r^{5}\right) .
$$

But $\left(R_{i j ; k}^{\tau}\right.$ stands for $R_{i j ; k}^{\tau}(0)$. No summation over $l$.)

$$
\begin{aligned}
R_{i j ; k}^{\tau} & \int_{S^{n}} x^{i} x^{j} x^{k} x^{l} d A \\
= & R_{l l ; l}^{\tau} \int_{S^{n}}\left(x^{l}\right)^{4} d A+\sum_{j, k \neq l} R_{l j ; k}^{\tau} \int_{S^{n}}\left(x^{l}\right)^{2} x^{j} x^{k} d A \\
& +\sum_{k, i \neq l} R_{i l ; k}^{\tau} \int_{S^{n}}\left(x^{l}\right)^{2} x^{i} x^{k} d A+\sum_{i, j \neq l} R_{i j ; l}^{\tau} \int_{S^{n}}\left(x^{l}\right)^{2} x^{i} x^{j} d A \\
& +\sum_{i, j, k \neq l} R_{i j ; k}^{\tau} \int_{S^{n}} x^{i} x^{j} x^{k} x^{l} d A \\
= & R_{l l ; l}^{\tau} \int_{S^{n}}\left(x^{l}\right)^{4} d A+\left(R_{l j ; j}^{\tau}+R_{i l ; i}^{\tau}+R_{i i ; l}^{\tau}\right) \int_{S^{n}}\left(x^{1}\right)^{2}\left(x^{2}\right)^{2} d A \\
& -3 R_{l l ; l}^{\tau} \int_{S^{n}}\left(x^{1}\right)^{2}\left(x^{2}\right)^{2} d A=\frac{2 \omega_{n+1}}{n+3} R_{, l}^{\tau},
\end{aligned}
$$

since $\int_{S^{n}}\left(x^{l}\right)^{4} d A=3 \int_{S^{n}}\left(x^{1}\right)^{2}\left(x^{2}\right)^{2} d A=2 \omega_{n+1} /(n+3)$ and $R_{l j ; j}^{\tau}=$ $R_{i l ; i}^{\tau}=\frac{1}{2} R_{, l}^{\tau}$ by the second Bianchi identity. Hence (1.7) follows.

LEMMA 1.3. Set

$$
H_{\varphi r}=\frac{\partial}{\partial r} H_{\varphi}, \quad H_{\varphi r r}=\frac{\partial^{2}}{\partial r^{2}} H_{\varphi} .
$$

We have for all $\tau$

(1) $H_{\varphi r}(0, \tau, 0)=0$,

(2) $H_{\varphi r r}(0, \tau, 0)$ is an even operator in the sense that if $\varphi$ is an even function, i.e. $\varphi(-x)=\varphi(x)$, then $H_{\varphi r r}(0, \tau, 0) \varphi$ is also an even function. 
Proof. Consider an arbitrary point $x_{0} \in S^{n}$. We may assume $x_{0}=$ $(0, \ldots, 0,1)$. Choose a local coordinate system $x=x(\theta)$ of $S^{n}$ around $x_{0}$ with $x(0)=x_{0}$, which is normal at $x_{0}$ w.r.t. the standard metric. Then

$$
\Delta_{r, \tau}=\frac{1}{\sqrt{h}} \frac{\partial \sqrt{h}}{\partial \theta_{i}} h^{i j} \frac{\partial}{\partial \theta_{j}}+\frac{\partial h^{i j}}{\partial \theta_{i}} \frac{\partial}{\theta_{j}}+h^{i j} \frac{\partial^{2}}{\partial \theta_{i} \partial \theta_{j}}
$$

where

$$
\begin{gathered}
\left(h^{i j}(\theta)\right)=\left(h_{i j}(\theta)\right)^{-1}, h(\theta)=\operatorname{det}\left(h_{i j}(\theta)\right) \text { and } \\
h_{i j}(\theta)=d s_{r, \tau}^{2}\left(\partial / \partial \theta_{i}, \partial / \partial \theta_{j}\right)
\end{gathered}
$$

(the dependence on $r, \tau$ is suppressed). Let the matrix $a(\theta)=\left(a_{i}^{j}(\theta)\right)$ be defined by the relation

$$
\left(\frac{\partial}{\partial \theta_{1}}, \ldots, \frac{\partial}{\partial \theta_{n}}, \frac{\partial}{\partial r}\right)=\left(\frac{\partial}{\partial x^{1}}, \ldots, \frac{\partial}{\partial x^{n+1}}\right) a(\theta) .
$$

Then we have

$$
a^{n+1}(0)=0, \quad 1 \leq i \leq n
$$

$$
\frac{\partial}{\partial \theta_{k}} a_{i}^{j}(0)=0, \quad 1 \leq i, j, k \leq n
$$

$$
h(\theta)=[\operatorname{det} a(\theta)]^{2} g^{\tau}(r x(\theta)) .
$$

Note that $(1.10)$ follows from the assumption that $x(\theta)$ is normal at $x_{0}=x(0)$. Since $\operatorname{det} a(\theta)=\left\|\frac{\partial}{\partial \theta_{1}} \wedge \cdots \wedge \frac{\partial}{\partial \theta_{n}}\right\|$, we also deduce from this assumption that

$$
\frac{\partial}{\partial \theta_{i}}(\operatorname{det} a)(0)=0, \quad 1 \leq i \leq n .
$$

From (1.10), (1.12) and the fact $\left(\partial g^{\tau} / \partial x^{k}\right)(0)=0,1 \leq k \leq n+1$ we immediately obtain

$$
\begin{gathered}
\left.\frac{\partial}{\partial r} h(0)\right|_{r=0}=0,\left.\quad \frac{\partial}{\partial \theta_{i}} h(0)\right|_{r=0}=0, \\
\left.\frac{\partial}{\partial r} \frac{\partial}{\partial \theta_{i}} h(0)\right|_{r=0}=0, \quad 1 \leq i \leq n .
\end{gathered}
$$

On the other hand, we have

$$
h_{i j}(\theta)=a_{i}^{k}(\theta) a_{j}^{l}(\theta) g_{k l}^{\tau}(r x(\theta))
$$


hence

$$
\left.\frac{\partial}{\partial r} h_{i j}(0)\right|_{r=0}=a_{i}^{k}(0) a_{j}^{l}(0) g_{k l, m}^{\tau}(0) x^{m}(0)=0
$$

$$
\begin{aligned}
\left.\frac{\partial}{\partial r} \frac{\partial}{\partial \theta_{k}} h_{i j}(0)\right|_{r=0}= & \frac{\partial}{\partial \theta_{k}}\left(a_{i}^{m} a_{j}^{l}\right)(0) g_{m l, m^{\prime}}^{\tau}(0) x^{m^{\prime}}(0) \\
& +a_{i}^{m}(0) a_{j}^{l}(0) g_{m l, m^{\prime}}^{\tau}(0) \frac{\partial x^{m^{\prime}}}{\partial \theta_{k}}(0)=0
\end{aligned}
$$

From (1.8), (1.15) and (1.16) one easily derives $\left.(\partial / \partial r) \Delta_{r, \tau}\right|_{r=0}=0$.

Next we compute $\left\|B_{r, \tau}\right\|^{2}$. Using the Gram-Schmidt orthogonalization procedure and the fact $g_{i j}^{\tau}(r x)=\delta_{i j}+O\left(r^{2}\right)$ we find orthonormal bases $e_{i}(r, \tau), 1 \leq i \leq n$, for $T_{x_{0}} S^{n}$ relative to $d s_{r, \tau}^{2}$ with $e_{i}(r, \tau)=\partial / \partial x^{i}+O\left(r^{2}\right)$. We compute the coefficients of the second fundamental form $B_{r, \tau}$

$$
b_{i j}^{r, \tau}=\nabla_{e_{i}(r, \tau)}^{r, \tau}\left(x^{k} \frac{\partial}{\partial x^{k}}\right) \cdot e_{j}(r, \tau),
$$

where $\nabla^{r, \tau}$ and the dot denote respectively the covariant differentiation and the inner product relative to the metric $d s_{r, \tau}^{2}$. Observe

$$
\nabla_{\partial / \partial x^{\prime}}^{r, \tau} \frac{\partial}{\partial x^{j}}=r^{(\tau)} \Gamma_{i j}^{k}(r x) \frac{\partial}{\partial x^{k}},
$$

where ${ }^{(\tau)} \Gamma_{i j}^{k}$ are the Christoffel symbols of the metric $d s^{2}$ of $M$ in the coordinates $\varphi_{\tau}$. Consequently

$$
\begin{aligned}
b_{i j}^{r, \tau} & =\nabla_{\partial / \partial x^{k}}^{r, \tau}\left(x^{k} \frac{\partial}{\partial x^{k}}\right) \cdot \frac{\partial}{\partial x^{j}}+O\left(r^{3}\right) \\
& =g_{i j}\left(r x_{0}\right)+r x_{0}^{k(\tau)} \Gamma_{k i}^{l}\left(r x_{0}\right) g_{l j}\left(r x_{0}\right)+O\left(r^{3}\right) \\
\left\|B_{r, \tau}\right\|^{2}\left(x_{0}\right)= & \sum_{1 \leq i, j \leq n} g_{i j}^{2}\left(r x_{0}\right) \\
& +2 r x_{0}^{k} \sum_{1 \leq i, j \leq n} g_{i j}\left(r x_{0}\right)^{(\tau)} \Gamma_{k i}^{l}\left(r x_{0}\right) g_{l j}\left(r x_{0}\right)+O\left(r^{3}\right) .
\end{aligned}
$$

It follows that $\left.(\partial / \partial r)\left\|B_{r, \tau}\right\|^{2}\left(x_{0}\right)\right|_{r=0}=0$. On the other hand it is easy to see that

$$
\operatorname{Ric}_{r, \tau}(\nu)\left(x_{0}\right)=r^{2} R_{i j}\left(r x_{0}\right) x_{0}^{i} x_{0}^{j} ;
$$

hence $\partial /\left.\partial r \operatorname{Ric}_{r, \tau}(\nu)\left(x_{0}\right)\right|_{r=0}=0$ and therewith the first part of the lemma is proved. 
Notice now $\left.\left(\partial^{2} / \partial r^{2}\right) \operatorname{Ric}_{r, \tau}(\nu)\left(x_{0}\right)\right|_{r=0}=2 R_{i j}(0) x_{0}^{i} x_{0}^{J}$. From $(1.17)$ we also get

$$
\begin{aligned}
\left.\frac{\partial^{2}}{\partial r^{2}}\left\|B_{r, \tau}\right\|^{2}\left(x_{0}\right)\right|_{r=0}= & 2 g_{i j}(0) g_{i j, k l}(0) x_{0}^{k} x_{0}^{l} \\
& +2 x_{0}^{k} \sum_{i \leq i, j \leq n} g_{i j}(0) \cdot{\frac{\partial}{\partial x^{m}}}^{(\tau)} \Gamma_{k i}^{l}(0) x_{0}^{m} g_{l j}(0) .
\end{aligned}
$$

Using $x=-x(\theta)$ as a coordinate system around $-x_{0}$ and performing the same computations as above we then deduce

$$
\begin{gathered}
\left.\frac{\partial^{2}}{\partial r^{2}} \operatorname{Ric}_{r, \tau}(\nu)\left(-x_{0}\right)\right|_{r=0}=\left.\frac{\partial^{2}}{\partial r^{2}} \operatorname{Ric}_{r, \tau}(\nu)\left(x_{0}\right)\right|_{r=0} \text { and } \\
\left.\frac{\partial^{2}}{\partial r^{2}}\left\|B_{r, \tau}\right\|^{2}\left(-x_{0}\right)\right|_{r=0}=\left.\frac{\partial^{2}}{\partial r^{2}}\left\|B_{r, \tau}\right\|^{2}\left(x_{0}\right)\right|_{r=0} .
\end{gathered}
$$

From (1.8), (1.9), (1.13), (1.14), (1.15) and (1.16) one obtains through elementary computations that at $x_{0}$ the operator

$$
\left.\left(\partial^{2} / \partial r^{2}\right) \Delta_{r, \tau}\right|_{r=0}
$$

equals

$$
\begin{aligned}
& -\left.\frac{1}{2} \frac{\partial^{2}}{\partial r^{2}} \frac{\partial}{\partial \theta_{i}} h(0)\right|_{r=0} \frac{\partial}{\partial \theta_{i}} \\
& \quad+\left(\left.\frac{\partial^{2}}{\partial r^{2}} A_{i j}(0)\right|_{r=0}-\left.\frac{\partial^{2}}{\partial r^{2}} h(0)\right|_{r=0} \delta_{i j}\right) \cdot \frac{\partial^{2}}{\partial \theta_{l} \partial \theta_{j}}
\end{aligned}
$$

where $A_{i j}$ denotes the cofactors of the matrix $\left(h_{i j}\right)$. But

$$
\begin{aligned}
& \frac{\partial^{2}}{\partial r^{2}}\left.\frac{\partial}{\partial \theta_{i}} h(0)\right|_{r=0} \\
& \quad=\left.\frac{\partial^{2}}{\partial r^{2}} \frac{\partial}{\partial \theta_{i}} g^{\tau}(r x(\theta))\right|_{\theta=0, r=0}=\frac{\partial^{2} g^{\tau}}{\partial x^{k} \partial x^{l}}(0) \frac{\partial x^{k}}{\partial \theta_{i}}(0) x^{l}(0)
\end{aligned}
$$

hence it is quadratic in $(x, D x)$, where $D x=\left(\partial x^{i} / \partial \theta_{j}\right)_{1 \leq i \leq n+1,1 \leq j \leq n}$. Easy computations also show that the coefficients of $\partial^{2} / \partial \theta_{l} \partial \theta_{J}$ above are quadratic in $(x, D x)$. Employing the coordinates $-x(\theta)$ around $-x_{0}$ we then see that $\left.\left(\partial^{2} / \partial r^{2}\right) \Delta_{r, \tau}\right|_{r=0}$ is an even operator. Since

$$
\left.\frac{\partial^{2}}{\partial r^{2}} \operatorname{Ric}_{r, \tau}(\nu)\right|_{r=0} \text { and }\left.\frac{\partial^{2}}{\partial r^{2}}\left\|B_{r, \tau}\right\|^{2}\right|_{r=0}
$$

have been shown to be even, the second part of the lemma is proved. 
DEFINITION 1.1. A smooth codimension 1 foliation $\mathscr{F}$ of $U \backslash\{p\}$ for a neighborhood $U$ of $p$ is called a foliation centered at $p$, provided that its leaves are all closed. If furthermore

$$
\sup _{S \in \mathscr{F}}\left(\sup _{S}\left\|B_{S}\right\| \operatorname{diam} S\right)<\infty
$$

where $B_{S}$ denotes the second fundamental form of $S$, then $\mathscr{F}$ is called a foliation regularly centered at $p$

THEOREM 1.1. If $p$ is a nondegenerate critical point of the scalar curvature function, then there exist $\delta>0$ and smooth functions $\tau=$ $\tau(r), \varphi=\varphi(r)$ with $\tau(0)=0$ such that $H\left(r, \tau(r), r^{2} \varphi(r)\right) \equiv n$ for $0 \leq r \leq \delta$. Hence the family $\mathscr{F}=\left\{S_{r}:=S_{r, \tau(r), r^{2} \varphi(r)}: 0 \leq r \leq \delta\right\}$ is a smooth family of constant mean curvature spheres with $S_{r}$ having mean curvature $n / r . \mathscr{F}$ is a foliation regularly centered at $p$. Moreover, $\mathscr{F}$ can actually be represented as $\left\{S_{r, 0, r \bar{\varphi}(r)}: 0<r \leq \delta\right\}$ for some smooth function $\bar{\varphi}(r)$. (In particular, each $S_{r}$ is a normal graph over $S_{r}(p)$.)

Proof. We first consider the equation $\widetilde{P}\left(H\left(r, \tau, r^{2} \varphi\right)\right)=0$. By Lemma 1.2 , Lemma 1.3 and the fact $\widetilde{P} L=0$ we can write this equation as follows (after division by $r^{3}$ )

$$
\begin{aligned}
& -\frac{\omega_{n+1}}{2(n+3)} R_{, l}^{\tau}(0) \mathbf{e}_{l}+O\left(r^{2}\right) \\
& +\widetilde{P}\left(r \int_{0}^{1} \int_{0}^{1} t H_{\varphi \varphi}\left(s r, \tau, s t r^{2} \varphi\right) \varphi \varphi d s d t\right. \\
& \quad+r \int_{0}^{1} \int_{0}^{1} \int_{0}^{1} s H_{\varphi r r}\left(u s r, \tau, u s t r^{2} \varphi\right) \varphi d u d s d t \\
& \quad+r^{2} \int_{0}^{1} \int_{0}^{1} \int_{0}^{1} s t H_{\varphi \varphi r}\left(u s r, \tau, u s t r^{2} \varphi\right) \\
& \quad \times 2 \varphi \varphi d u d s d t)=0
\end{aligned}
$$

where

$$
H_{\varphi \varphi}(r, \tau, \psi) \varphi \varphi^{\prime}=\left.\frac{d}{d t} H_{\varphi}\left(r, \tau, \psi+t \varphi^{\prime}\right) \varphi\right|_{t=0},
$$

and $H_{\varphi \varphi r}=(\partial / \partial r) H_{\varphi \varphi}$. By the assumption, $\left.R_{, l}^{\tau}(0)\right|_{\tau=0}=0$ and the Hessian matrix

$$
\left(\left.\frac{\partial}{\partial \tau_{k}} R_{, l}^{\tau}(0)\right|_{\tau=0}\right)_{k, l}
$$


is nonsingular. Applying the implicit function theorem we obtain a solution $\tau=\tau(r, \varphi)$ of the equation $\widetilde{P}\left(H\left(r, \tau, r^{2} \varphi\right)\right)=0$ around $r=0, \varphi=\varphi_{0}$ with $\tau\left(0, \varphi_{0}\right)=0$, where $\varphi_{0} \in K^{\perp}$ is determined by the relation $L \varphi_{0}=\frac{1}{3} R_{i j}(0) x^{i} x^{j} \quad\left(R_{i j}(0) x^{i} x^{j}\right.$ denotes the function defined on $S^{n}$ whose value at $x \in S^{n}$ is given by the quadratic form $\left.R_{i j}(0) x^{i} x^{j}\right)$.

Now we consider the mapping $(r, \varphi) \mapsto H\left(r, \tau(t, \varphi), r^{2} \varphi\right)$ whose values lie in $K^{\perp}$ by the construction of $\tau(r, \varphi)$. We restrict $\varphi$ to be in $K^{\perp}$. From (1.5) and Lemma 1.3 we obtain (for small $r, \tau, \varphi$ )

$$
\begin{aligned}
H(r, \tau, \varphi)= & n-\frac{1}{3} R_{i j}^{\tau}(0) x^{i} x^{j} r^{2} \\
& -\frac{1}{4} R_{i j ; k}^{\tau}(0) x^{i} x^{j} x^{k} r^{3}+O\left(r^{4}\right) \\
& +\int_{0}^{1} \int_{0}^{1} t H_{\varphi \varphi}(s r, \tau, s t \varphi) \varphi \varphi d s d t \\
& +r^{2} \int_{0}^{1} \int_{0}^{1} \int_{0}^{1} s H_{\varphi r v}(u s r, \tau, u s t \varphi) \varphi d u d s d t \\
& +r \int_{0}^{1} \int_{0}^{1} \int_{0}^{1} s t H_{\varphi \varphi r}(u s r, \tau, u s t \varphi) \varphi \varphi d u d s d t \\
& +L \varphi
\end{aligned}
$$

By the implicit function theorem we can solve $H\left(t, \tau(r, \varphi), r^{2} \varphi\right)=n$ after dividing it out by $r^{2}$ to get $\varphi=\varphi(r), 0<r \leq \delta$ for some $\delta>0$ with $\varphi(0)=\varphi_{0}$.

Intermediate Remark. Define a map $W$ from a neighborhood of $\left(0,0, \varphi_{0}\right) \in \mathbb{R} \times \mathbb{R}^{n+1} \times K^{\perp}$ into $\mathbb{R}^{n+1} \times L\left(K^{\perp}\right)$ as follows

$$
W(r, \tau, \varphi)=\left(\frac{1}{r^{3}} \widetilde{P}\left(H\left(r, \tau, r^{2}, \varphi\right)\right), \frac{1}{r^{2}} P^{\perp}\left(H\left(r, \tau, r^{2} \varphi\right)-n\right)\right),
$$

where $P^{\perp}$ denotes the $L_{2}$ orthogonal projection from $C^{0,1 / 2}\left(S^{n}\right)$ onto $L\left(K^{\perp}\right)$. Then $W(r, \tau(r), \varphi(r)) \equiv 0$ and the implicit function theorem implies: for $(r, \tau, \varphi) \in \mathbb{R} \times \mathbb{R}^{n+1} \times K^{\perp}$ with $r, \tau$ and $\left\|\varphi-\varphi_{0}\right\|$ sufficiently small we have $\tau=\tau(r), \varphi=\varphi(r)$, provided that $W(r, \tau, \varphi)=0$.

It is clear that the family $\left\{S_{r}=S_{r, \tau(r), r^{2} \varphi(r)}: 0<r \leq \delta\right\}$ with $\tau(r)=\tau(r, \varphi(r))$ is a smooth family of embedded constant mean curvature spheres with $S_{r}$ having mean curvature $n / r$. Geometrically, we obtained this family by moving the center $p$ of the geodesic spheres $S_{r}(p)$ to $\exp _{p}\left(\tau^{i}(r) e_{i}\right)$ and then performing the normal perturbation $r^{3} \varphi(r)$. In order to show that this family constitutes a foliation, we 
need to be sure that the new centers are sufficiently close to $p$. From (1.18) we see that

$$
\tau(r)=O\left(r^{2}\right)
$$

whenever the following is true

$$
\widetilde{P}\left(H_{\varphi \varphi}(0, \tau, 0) \varphi_{0} \varphi_{0}\right)=0, \quad \widetilde{P}\left(H_{\varphi r r}(0, \tau, 0) \varphi_{0}\right)=0 .
$$

To show (1.21) we first consider the equation

$$
\Delta_{S^{n}} \varphi_{0}+n \varphi_{0}=\frac{1}{3} R_{i j}(0) x^{i} x^{j} .
$$

By the unique solvability of this equation under the constraint $\varphi_{0} \in$ $K^{\perp}$ and the invariance of the Laplacian under isometries we deduce that $\varphi_{0}$ is even. Lemma 1.3 then implies the second equation in (1.21). On the other hand, we conclude that the mean curvature functions $H\left(0,0, \tau \varphi_{0}\right)$ of the hypersurfaces $S_{t \varphi_{0}}^{n}$ (in the euclidean metric) are even. Since $H_{\varphi \varphi}(0, \tau, 0) \varphi_{0} \varphi_{0}=H_{\varphi \varphi}(0,0,0) \varphi_{0} \varphi_{0}=$ $\left.\left(\partial^{2} / \partial t^{2}\right) H\left(0,0, t \varphi_{0}\right)\right|_{t=0}$, the first equation in (1.21) also follows and therewith (1.20) is established.

Now we define $\Psi^{r}=\exp _{p}^{-1} \exp _{c(\tau(r))}, \psi(r, x)=\Psi^{r}\left(r\left(x+r^{2} \varphi(r)(x)\right)\right)$ and $\beta(r, x)=\psi /\|\psi\|$ if $\|\psi(r, x)\| \neq 0$, where $x \in S^{n}$.

Claim. For sufficiently small $r>0,\|\psi(r, x)\|$ is nonzero for all $x \in S^{n}$ and $\beta(r, \cdot)$ is a diffeomorphism from $S^{n}$ onto itself. Moreover, $\beta(r, x)$ extends smoothly to $r=0$ with $\beta(0, \cdot)=$ the identity.

In fact, we have

$$
\begin{gathered}
\frac{\partial \psi}{\partial r}=\left(d_{x} \Psi^{r}\right)\left(x+r^{2} \varphi(r)(x)+r\left(r^{2} \varphi(r)(x)\right)_{r}\right) \\
+\left(\frac{\partial \Psi r}{\partial r}\right)\left(r\left(x+r^{2} \varphi(r)(x)\right)\right) \\
\left.\frac{\partial \Psi r}{\partial r}\right|_{r=0}=\left.\left.\frac{\partial}{\partial \tau^{i}}\left(\exp _{p}^{-1} \exp _{c(\tau)}\right)\right|_{\tau=0} \frac{\partial \tau^{i}}{\partial r}\right|_{r=0}=0
\end{gathered}
$$

by $(1.19)$, hence $(\partial \psi / \partial r)(0, x)=x$. It follows that $\psi(r, x)=r x+$ $O\left(r^{2}\right)$. Consequently $\psi(r, x) \neq 0$ for sufficiently small $r>0$ and $\beta(r, x)=(x+O(r)) /\|x+O(r)\|$. Clearly $\beta$ extends smoothly to $r=0$ with $\beta(0, \cdot)=$ the identity. For $r$ near $0, \beta(r, \cdot)$ then is a diffeomorphism. 
Now set $\varphi(r, x)=\left\|\psi\left(r, \beta^{-1}(r, x)\right)\right\|$, where $\beta^{-1}(r, \cdot)$ denotes the inverse of $\beta(r, \cdot)$. We have

$$
\begin{aligned}
\frac{\partial \varphi}{\partial r} & =\frac{1}{\|\psi\|} \psi \cdot\left(\frac{\partial \psi}{\partial r}+\left(d_{x} \psi\right)\left(\frac{\partial}{\partial r} \beta^{-1}\right)\right) \\
& =\frac{1}{\|x+O(r)\|}(x+O(r)) \cdot(x+O(r))
\end{aligned}
$$

whence $\left.(\partial \varphi / \partial r)\right|_{r=0}=1$. We conclude that $\varphi(r, x)$ is strictly increasing w.r.t. $r$ for $r$ small and hence $\widetilde{S}_{r}, \widetilde{S}_{r^{\prime}}$ are disjoint for small $r, r^{\prime}$ with $r \neq r^{\prime}$. The family $\left\{S_{r}\right\}_{0<r \leq \delta}$ therefore constitutes a foliation if $\delta$ is chosen small enough. It is easy to see that this foliation is regularly centered at $p$. The verification of the last statement of the theorem is also straightforward.

2. Uniqueness and nonexistence. Let $\mathscr{F}$ be a foliation regularly centered at $p$, whose leaves have constant mean curvature.

\section{LEMMA 2.1. The following holds:}

(1) There is a neighborhood $\Omega$ of $p$ together with a constant $c>1$ such that the absolute value of the mean curvature of $S$ lies in the interval $(1 / c \operatorname{diam} S, c / \operatorname{diam} S)$, provided that $S$ is a leaf of $\mathscr{F}$ and $S \subset \Omega$;

(2) $\operatorname{diam} S \rightarrow 0$ as $\operatorname{dist}(p, s) \rightarrow 0$ for leaves $S$ of $\mathscr{F}$;

(3) each leaf of $\mathscr{F}$ bounds a domain containing $p$;

(4) the leaves of $\mathscr{F}$ can be parametrized as a smooth family $S_{t}$, $0<t \leq 1$ with $S_{t} \neq S_{t^{\prime}}$ if $t \neq t^{\prime}$ and $\lim _{t \rightarrow 0} \operatorname{diam} S_{t}=0$.

Proof. (1) Choose $\Omega$ such that $\operatorname{dist}^{2}(p, \cdot)$ is smooth and strictly convex on $\bar{\Omega}$. For $S \in \mathscr{F}, S \subset \Omega$ let $\rho$ denote the restriction of this function to $S$. At a maximum point we have $\Delta \rho \leq 0$, which by simple computations implies the desired lower bound. The upper bound follows from Definition 1.1.

(2) This is a simple consequence of the assumption that all leaves of $\mathscr{F}$ are closed.

(3) and (4) Consider a leaf $S$ sufficiently close to $p$. Clearly $S$ bounds a domain. But a compact manifold with nonempty connected boundary cannot be smoothly foliated by closed leaves, whence this domain contains $p$. By a continuity argument one then derives (3) 
and that the leaf topology of $\mathscr{F}$ is equivalent to $(0,1]$. The last fact implies (4).

LEMMA 2.2. For $t$ sufficiently small, $S_{t}$ is unstable, i.e. the first eigenvalue of the Jacobi operator $\Delta+\|B\|^{2}+\operatorname{Ric}(\nu)$ on $S_{t}$ is negative. Consequently, $H^{\prime}(t) \neq 0$, where $H(t)$ denotes the inward mean curvature of $S_{t}$.

Proof. Consider a sequence $t_{k} \rightarrow 0$ and write $S_{t_{k}}$ as $S_{k}$. Define $\widetilde{S}_{k}=\alpha_{1 / d\left(S_{k}\right)}\left(\varphi_{0}\left(S_{k}\right)\right)$. Then the diameter of $\widetilde{S}_{k}$ relative to the metric $d s_{0, d\left(S_{k}\right)}^{2}$ is 1 . Since $d s_{0, d\left(S_{k}\right)}^{2}$ converges to the euclidean metric as $k \rightarrow \infty$ and each $\widetilde{S}_{k}$ encloses the origin, we may assume $\widetilde{S}_{k} \subset \mathbb{B}_{2}$ for all $k$. Then the second fundamental form bound postulated in Definition 1.1 and the standard elliptic PDE theory imply that local pieces of $\widetilde{S}_{k}$ subconverge smoothly. Consequently one of the following two cases occurs: (i) (after selection of a subsequence of $S_{k}$ ) there are pieces $\widetilde{S}_{k}^{\prime}, \widetilde{S}_{k}^{\prime \prime}$ of $\widetilde{S}_{k}$ such that $\widetilde{S}_{k}^{\prime}$ and $\widetilde{S}_{k}^{\prime \prime}$ converge to the same piece of surface, while the outer unit normal approaches opposite directions. This implies that the mean curvature of $\widetilde{S}_{k}$ relative to $d s_{0, d\left(S_{k}\right)}^{2}$ converges to zero. (ii) A subsequence $\widetilde{S}_{k^{\prime}}$ converges smoothly to an immersed, closed hypersurface $S$ of euclidean constant mean curvature, which is the boundary of a domain. The classical argument of Alexandrov shows that $S$ is a round sphere. By Lemma 2.2, case (1) cannot happen. Since round spheres are unstable in the euclidean metric (the first eigenvalue of the Jacobi operator on a round sphere of radius 1 is $-n), \widetilde{S}_{k^{\prime}}$ is unstable whenever $k^{\prime}$ is large enough. Since the sequence $t_{k}$ is arbitrary, we conclude that for $t$ sufficiently small, $S_{t}$ is unstable.

Now assume $H^{\prime}(t)=0$. Representing $S_{t^{\prime}}$ as normal graphs over $S_{t}$ for $t^{\prime}$ near $t$, we derive that the Jacobi operator on $S_{t}$ has a positive eigenfunction with eigenvalue 0 . This would imply that $S_{t}$ is stable.

Since $H^{\prime}(t) \neq 0$, we can introduce a new parameter $r=n / H(t)$. Then we obtain a parametrization $S_{r}, 0<r \leq r_{0}$ (with some $r_{0}>0$ ) for the leaves of (a restriction of) $\mathscr{F}$ with the property that the inward mean curvature of $S_{r}$ equals $n / r$. We put $\widetilde{S}_{r}=\alpha_{1 / r}\left(\varphi_{0}^{-1}\left(S_{r}\right)\right)$ and $x(r)=$ the center of mass for $\widetilde{S}_{r}$.

Let $S^{n}(a)$ denote the unit sphere of a center $a$ in $\mathbb{R}^{n+1}$. For a smooth function $\varphi$ on $S^{n}(a), S^{n}(a)_{\varphi}$ is defined in a similar way to $S_{\varphi}^{n}$. 
LEMMA 2.3. There exists some $\bar{r}_{0}>0$ such that for each $r \in$ $\left(0, \bar{r}_{0}\right), \quad \widetilde{S}_{r}=S^{n}(x(r))_{\bar{\varphi}(r)}$ for a smooth function $\bar{\varphi}(r)$ on $S^{n}(x(r))$ with $\lim _{r \rightarrow 0}\|\bar{\varphi}(r)\|_{C^{3}}=0$. (It follows automatically that $\bar{\varphi}(r)$ depends smoothly on $r$.)

Proof. Assume the contrary. Then there is a sequence $r_{k} \rightarrow 0$ together with a number $\delta>0$ such that for each $k$, either $\widetilde{S}_{r_{k}}$ is not a smooth normal graph over $S^{n}\left(x\left(r_{k}\right)\right)$ or $\widetilde{S}_{r_{k}}=S^{n}\left(x\left(r_{k}\right)\right)_{\varphi_{k}}$ for a function $\varphi_{k}$ with $\left\|\varphi_{k}\right\|_{C^{3}} \geq \delta$. Note that $\varphi_{k}$ is uniquely determined and the mean curvature of $\widetilde{S}_{r}$ in the metric of $d s_{0, r}^{2}$ equals $n$. By the arguments in the proof of Lemma 2.2 we can find a subsequence $\widetilde{S}_{r_{k}}$ which converges smoothly to a round sphere of radius 1 . For $k^{\prime}$ large we then have $\widetilde{S}_{r_{k^{\prime}}}=S^{n}\left(x\left(r_{k^{\prime}}\right)\right)_{\varphi_{k^{\prime}}}$ with $\left\|\varphi_{k^{\prime}}\right\|_{C^{3}}<\delta$, a contradiction.

Corollary 2.1. There are $r_{1}>0, \delta>0$ such that for $r \leq r_{1}$ and $\|a\| \leq \delta, \alpha_{1 / r}\left(\varphi_{r(x(r)+a)}^{-1}\left(S_{r}\right)\right)$ is a smooth normal graph over $S^{n}$, thus $S_{r}=S_{r, r(x(r)+a), \varphi(r, a)}$ for a smooth function $\varphi(r, a)$ on $S^{n}$. We have $\|\varphi(r, a)\|_{C^{3} \rightarrow 0}$ as $r \rightarrow 0, a \rightarrow 0$.

Proof. Firstly, there are $r_{1}>0, \delta>0$ such that for $r \leq r_{1},\|a\| \leq$ $\delta, \widetilde{S}_{r}$ is a smooth (euclidean) normal graph over $S^{n}(a+r(r))$, thus $\widetilde{S}_{r}=S^{n}(a+x(r))_{\bar{\varphi}(r, a)}$ for a smooth function $\bar{\varphi}(r, a)$ on $S^{n}(a+x(r))$. Since the metric $d s_{0, r}^{2}$ approaches the euclidean metric as $r \rightarrow 0$, for $r$ small enough (and $\|a\| \leq \delta), \widetilde{S}_{r}$ will be a smooth (geodesic) normal graph over the geodesic sphere $S_{*}^{n}(a+x(r))$ of radius 1 and center $a+x(r)$ relative to $d s_{0, r}^{2}$. We denote the defining function of this normal graph relative to the inward normal by $\bar{\varphi}(r, a)$. It follows that $S_{r}$ is a smooth normal graph over $S_{r}\left(\varphi_{0}(r(a+x(r)))\right)$, which in turn implies $S_{r}=S_{r, r(a+x(r)), \varphi(r, a)}$. We can relate $\varphi(r, a)$ to $\bar{\varphi}(r, a)$ as follows. Denote by $\psi_{r, a}$ the exponential map at $a+x(r)$ relative to the metric $d s_{0, r}^{2}$. Then $\varphi(r, a)(x)=\bar{\varphi}(r, a)\left(\psi_{r, a}\left(x^{i} e_{i}^{*}\right)\right)$, where $e_{i}^{*}=\left(\varphi_{0}^{-1}\right)_{*}\left(e_{i}^{r(a+x(r))}\right)$. The last statement of the corollary follows easily.

Next we want to compute the dependence of $\varphi(r, a)$ on $a$. Let us consider a hypersurface $S$ which is a smooth normal graph over $S^{n}\left(a_{0}\right)$ as well as $S^{n}\left(a_{0}+a\right)$, thus $S=S^{n}\left(a_{0}\right) \varphi=S^{n}\left(a_{0}+a\right)_{\varphi^{\prime}}$ for some $\varphi, \varphi^{\prime}$. Given $x \in S^{n}\left(a_{0}\right)$, we consider the point $y \in S$ above 
$x, y=a_{0}+(1-\varphi(x))\left(x-a_{0}\right)$. For a unique point $x^{\prime} \in S^{n}\left(a_{0}+a\right)$ we also have $y=a_{0}+a+\left(1-\varphi^{\prime}\left(x^{\prime}\right)\right)\left(x^{\prime}-a_{0}-a\right)$. It follows that

$$
\begin{gathered}
x^{\prime}=F(x):=a+a_{0}+\frac{(1-\varphi(x))\left(x-a_{0}\right)-a}{\left\|(1-\varphi(x))\left(x-a_{0}\right)-a\right\|}, \\
\varphi^{\prime}\left(x^{\prime}\right)=1-\left\|(1-\varphi(x))\left(x-a_{0}\right)-a\right\| .
\end{gathered}
$$

We consider the projection of $\varphi^{\prime}$ onto the spherical harmonics on $S^{n}\left(a_{0}+a\right)$,

$$
\begin{aligned}
\frac{1}{\omega_{n+1}} & \int_{S^{n}\left(a_{0}+a\right)} \varphi^{\prime}\left(x^{\prime}\right)\left(x^{\prime}-a_{0}-a\right) d A \\
= & \frac{1}{\omega_{n+1}} \int_{S^{n}\left(a_{0}\right)}\left(1-\left\|(1-\varphi(x))\left(x-a_{0}\right)-a\right\|\right) \\
& \cdot \frac{(1-\varphi(x))\left(x-a_{0}\right)-a}{\left\|(1-\varphi(x))\left(x-a_{0}\right)-a\right\|} J_{F}(x) d A,
\end{aligned}
$$

where $J_{F}$ denotes the Jacobian of the map $F: S^{n}\left(a_{0}\right) \rightarrow S^{n}\left(a_{0}+a\right)$. Elementary computations show (for $a,\|\varphi\|_{C^{1}}$ sufficiently small)

$$
\begin{aligned}
& \frac{1}{\omega_{n+1}} \int_{S^{n}\left(a_{0}+a\right)} \varphi^{\prime}\left(x^{\prime}\right)\left(x^{\prime}-a_{0}-a\right) d A \\
& \quad=\frac{1}{\omega_{n+1}} \int_{S^{n}\left(a_{0}\right)} \varphi(x)\left(a-a_{0}\right) d A+a+B_{a, \varphi}(a, \varphi),
\end{aligned}
$$

where $B_{a, \varphi}: \mathbb{R}^{n+1} \times C^{1}\left(S^{n}\left(a_{0}\right)\right) \rightarrow \mathbb{R}^{n+1}$ stands for a continuous bilinear form depending smoothly on $a, \varphi$.

LEMMA 2.3. There is a smooth function $a(r), 0<r \leq r_{1}^{\prime}$ for some $r_{1}^{\prime}>0$ such that $\lim _{r \rightarrow 0} a(r)=0$ and $P(\varphi(r, a(r)))=0$ for every $r$.

Proof. Define a map $G(r, a)=\widetilde{P}(\varphi(r, a))$. We have

$$
\begin{aligned}
G(r, a) & =\frac{1}{\omega_{n+1}} \int_{S^{n}} \varphi(r, a)(x) x d A \\
& =\frac{1}{\omega_{n+1}} \int_{S^{n}} \bar{\varphi}(r, a)\left(\psi_{r, a}\left(x^{i} e_{i}^{*}\right)\right) x d A .
\end{aligned}
$$

Note the following fact: as $r \rightarrow 0$ (with $|a| \leq \delta$ ), the geodesic sphere $S_{*}^{n}(a+x(r))$ and the euclidean sphere $S^{n}(a+x(r))$ approach each other smoothly and all geometric quantities measured in $d s_{0, r}^{2}$ con. verge to those measured in the euclidean metric. Hence $\varphi(r, a) \circ Q_{r}$ and $\bar{\varphi}(r, a)$ approach each other smoothly as $r \rightarrow 0$, where $Q_{r}$ denotes the radial projection from $S^{n}(a+x(r))$ onto $S_{*}^{n}(a+x(r))$. Besides, the mappings $x \mapsto \psi_{r, a}\left(x^{i} e_{r}^{*}\right)$ and $x \mapsto a+x(r)+x$ approach 
each other as $r \rightarrow 0$. We conclude

$$
\begin{aligned}
G(r, a) & =\frac{1}{\omega_{n+1}} \int_{S^{n}} \bar{\varphi}(r, a)(a+x(r)+x) x d A+u(r)(a) \\
& =\frac{1}{\omega_{n+1}} \int_{S^{n}(a+x(r))} \bar{\varphi}(r, a)(x)(x-a-x(r)) d A+u(r)(a),
\end{aligned}
$$

where $u(r)$ denotes a smooth map depending smoothly on $r>0$ with $\|u(r)\|_{C^{1}\left(\mathbb{B}_{\delta}\right)} \rightarrow 0$ as $r \rightarrow 0$. Applying (2.3) we then get $(\bar{\varphi}(r)$ was defined in Lemma 2.3)

$$
\begin{aligned}
G(r, a)= & \frac{1}{\omega_{n+1}} \int_{S^{n}(x(r))} \bar{\varphi}(r)(x)(x-x(r)) d A \\
& +a+B_{a, \varphi(r)}(a, \bar{\varphi}(r))+u(r)(a) .
\end{aligned}
$$

By the inverse mapping theorem and the properties of $B_{a, \varphi}, u(r)$ and $\bar{\varphi}(r)$, there are $r_{1}^{\prime}>0, \delta^{\prime}>0$ and a natural number $m$ such that for $r<r_{1}^{\prime}, G(r, \cdot)$ is a diffeomorphism from $\mathbb{B}_{\delta^{\prime}}$ onto a domain containing $\mathbb{B}_{\delta^{\prime} / m}$. Hence we get a unique solution $a(r)$ of the equation $G(r, a)=0$. Clearly $a(r) \rightarrow 0$ as $r \rightarrow 0$.

Henceforth we put $\tau_{1}(r)=r(x(r)+a(r)), \varphi^{*}(r)=\varphi(r, a(r))$. We have obtained a nice parametrization $S_{r}=S_{r, \tau(r), \varphi^{*}(r)}$ for the leaves of (a restriction of) $\mathscr{F}$. The crucial property is $P(\varphi(r))=0$, $\lim _{r \rightarrow 0} \tau_{1}(r)=0$ and $\lim _{r \rightarrow 0}\left\|\varphi^{*}(r)\right\|_{C^{3}}=0$.

We shall call a smooth codimension 1 foliation with constant mean curvature leaves a constant mean curvature foliation.

THEOREM 2.1. (1) If $p$ is not a critical point of the scalar curvature function, then there exists no constant mean curvature foliation regularly centered at $p$.

(2) Assume that $p$ is a nondegenerate critical point of the scalar curvature function and let $\mathscr{F}_{0}$ be the constant mean curvature foliation obtained in Theorem 1.1. If $\mathscr{F}$ is a constant mean curvature foliation regularly centered at $p$, then either $\mathscr{F}_{0}$ is a restriction of $\mathscr{F}$ or $\mathscr{F}$ is a restriction of $\mathscr{F}_{0}$.

Proof. Let $\mathscr{F}$ be a constant mean curvature foliation regularly centered at $p$ and $S_{r}=S_{r, \tau_{1}(r), \varphi^{*}(r)}$ the parametrization obtained above. Since the mean curvature of $S_{r}$ is $n / r$, we have $H\left(r, \tau_{1}(r), \varphi^{*}(r)\right)=$ $n$ and hence

$$
P^{\perp}\left(H\left(r, \tau_{1}(r), \varphi^{*}(r)\right)-n\right)=0,
$$




$$
\widetilde{P}\left(H\left(r, \tau_{1}(r), \varphi^{*}(r)\right)\right)=0 .
$$

From (2.5) and (1.19) we derive $(L+A(r)) \varphi^{*}(r)=b\left(r, \tau_{1}\right) r^{2}$, where $b(r, \tau) \in K^{\perp}$ denotes a smooth function of $r$ and $\tau, A(r): K^{\perp} \rightarrow$ $L\left(K^{\perp}\right)$ denotes a linear operator with $\lim _{r \rightarrow 0}\|A(r)\|=0$ and $K^{\perp}$, $L\left(K^{\perp}\right)$ are endowed with $C^{2,1 / 2}$ and $C^{0,1 / 2}$ norms respectively. We immediately deduce $\left\|\varphi^{*}(r)\right\|_{C^{2,1 / 2}} \leq c r^{2}$ for a constant $c$. Now define $\varphi_{1}(r)=\varphi^{*}(r) / r^{2}$. Inserting $\tau=\tau_{1}(r)$ and $\varphi=r^{2} \varphi_{1}(r)$ into (1.19), dividing the resulting equation by $r^{2}$ we easily deduce that $\varphi^{*}(r)$ converges to $\varphi_{0}$ as $r$ goes to zero. By the Intermediate Remark in the proof of Theorem 1.1 we conclude that $\tau_{1}(r)=\tau(r)$ and $\varphi_{1}(r)=$ $\varphi(r)$ for $r$ small enough, provided that $p$ is a nondegenerate critical point of the scalar curvature function. This finishes the proof of the theorem.

\section{REFERENCES}

[1] T. Aubin, Nonlinear Analysis on Manifolds, Monge-Ampère Equations, Springer, New York, 1982.

[2] J. Lee and T. Parker, The Yamabe problem, Bull. Amer. Math. Soc., 17 (1987), 37-81.

Received April 18, 1989. Research partially supported by an NSF grant.

STANFORD UNIVERSITY

STANFORD, CA 94305-2125 


\title{
PACIFIC JOURNAL OF MATHEMATICS EDITORS
}

\author{
V. S. VARADARAJAN \\ (Managing Editor) \\ University of California \\ Los Angeles, CA 90024-1555-05 \\ Herbert Clemens \\ University of Utah \\ Salt Lake City, UT 84112 \\ THOMAS ENRIGHT \\ University of California, San Diego \\ La Jolla, CA 92093
}

R. FINN

Stanford University

Stanford, CA 94305

Hermann FlaschKa

University of Arizona

Tucson, AZ 85721

VAUGHaN F. R. JoNes

University of California

Berkeley, CA 94720

Steven Kerckhoff

Stanford University

Stanford, CA 94305

\section{C. MOORE}

University of California

Berkeley, CA 94720

Martin ScharlemanN

University of California

Santa Barbara, CA 93106

HAROLD STARK

University of California, San Diego

La Jolla, CA 92093
R. ARENS
E. F. BECKENBACH
F. WolF
K. YoshidA

\section{ASSOCIATE EDITORS}

\begin{tabular}{ll}
\multicolumn{1}{c}{ SUD-1982) } & \multicolumn{1}{c}{$(1904-1989)$} \\
UNIVERSITY OF ARIZONA & UNIVERSITY OF OREGON \\
UNIVERSITY OF BRITISH COLUMBIA & UNIVERSITY OF SOUTHERN CALIFORNIA \\
CALIFORNIA INSTITUTE OF TECHNOLOGY & STANFORD UNIVERSITY \\
UNIVERSITY OF CALIFORNIA & UNIVERSITY OF HAWAII \\
MONTANA STATE UNIVERSITY & UNIVERSITY OF TOKYO \\
UNIVERSITY OF NEVADA, RENO & UNIVERSITY OF UTAH \\
NEW MEXICO STATE UNIVERSITY & WASHINGTON STATE UNIVERSITY \\
OREGON STATE UNIVERSITY & UNIVERSITY OF WASHINGTON \\
\hline
\end{tabular}

The Supporting Institutions listed above contribute to the cost of publication of this Journal, but they are not owners or publishers and have no responsibility for its content or policies.

\begin{abstract}
Mathematical papers intended for publication in the Pacific Journal of Mathematics should be in typed form or offset-reproduced (not dittoed), double spaced with large margins. Please do not use built up fractions in the text of the manuscript. However, you may use them in the displayed equations. Underline Greek letters in red, German in green, and script in blue. The first paragraph must be capable of being used separately as a synopsis of the entire paper. In particular it should contain no bibliographic references. Please propose a heading for the odd numbered pages of less than 35 characters. Manuscripts, in triplicate, may be sent to any one of the editors. Please classify according to the 1980 Mathematics Subject Classification (1985 Revision) scheme which can be found in the December index volumes of Mathematical Reviews. Supply name and address of author to whom proofs should be sent. All other communications should be addressed to the managing editor, or Elaine Barth, University of California, Los Angeles, California 90024-1555-05.

There are page-charges associated with articles appearing in the Pacific Journal of Mathematics. These charges are expected to be paid by the author's University, Government Agency or Company. If the author or authors do not have access to such Institutional support these charges are waived. Single authors will receive 50 free reprints; joint authors will receive a total of 100 free reprints. Additional copies may be obtained at cost in multiples of 50 .
\end{abstract}

The Pacific Journal of Mathematics (ISSN 0030-8730) is published monthly except for July and August. Regular subscription rate: $\$ 190.00$ a year (10 issues). Special rate: $\$ 95.00$ a year to individual members of supporting institutions.

Subscriptions, orders for numbers issued in the last three calendar years, and changes of address should be sent to Pacific Journal of Mathematics, P.O. Box 969, Carmel Valley, CA 93924, U.S.A. Old back numbers obtainable from Kraus Periodicals Co., Route 100, Millwood, NY 10546.

The Pacific Journal of Mathematics at P.O. Box 969, Carmel Valley, CA 93924 (ISSN 0030-8730) is published monthly except for July and August. Second-class postage paid at Carmel Valley, California 93924, and additional mailing offices. Postmaster: send address changes to Pacific Journal of Mathematics, P.O. Box 969, Carmel Valley, CA 93924.

PUBLISHED BY PACIFIC JOURNAL OF MATHEMATICS, A NON-PROFIT CORPORATION Copyright (C) 1991 by Pacific Journal of Mathematics 